\title{
Arquitetura da informação em repositórios institucionais: desafios e perspectivas
}

\author{
Edilene Maria Silva* \\ Marckson Roberto Ferreira de Sousa \\ Samuel Alves Monteiro
}

Artículo recibido:

13 de agosto de 2015

Artículo aceptado:

16 de noviembre de 2016

\section{RESUMEN}

El acceso a la información científica provoca acciones dirigidas por las tecnologías de la información y la comunicación. Los repositorios, que reúnen la producción científica de una institución, deben estar alineados a los principios establecidos por la arquitectura de la información como forma de ser más eficientes y eficaces. Este estudio presenta el análisis del repositorio institucional de la Universidad Federal de Brasilia, objetivando verificar si éste emplea los principios propuestos por Morville y Rosenfeld. El repositorio analizado presenta compatibilidad con algunos principios, si bien necesita solucionar deficiencias referentes

* Universidade Federal da Paraíba, Brasil.

dilemabr@gmail.com

marckson.dci.ufpb@gmail.com samuelmonteiro00@yahoo.com.br

INVESTIGACIÓN BIBLIOTECOLÓGICA, vol. 32, Núm. 76, julio/septiembre, 2018, México, ISSN: 2448-8321 pp. 45-61 
al sistema de búsqueda, accesibilidad y atención de las demandas de los usuarios.

Palabras clave: Repositorio Institucional; Arquitectura de la Información; Acceso a la Información Digital.

Information architecture in institutional repositories: Challenges and perspectives

Edilene Maria-Silvia, Marckson-Roberto Ferreira-deSousa and Samuel Alves-Monteiro

\begin{abstract}
Concerning with the access to scientific information drives actions by information and communication technologies. The repositories, which gather all the scientific production of an institution, have to be aligned to the principles established by the Information Architecture as a way to be more efficient and effective. This study presents the analysis of the institutional repository of the Federal University of Brasília in order to verify if it uses the principles proposed by Morville and Rosenfeld. This repository presents compatibility with some principles; however, it needs to solve problems regarding the system of search, accessibility, and service to the users' demands.
\end{abstract}

Keywords: Institutional Repository; Information Architecture; Access to Digital Information.

\title{
INTRODUÇÃO
}

Significativa mobilização está sendo realizada pela comunidade científica Onas instituições brasileiras de ensino superior (IES) em prol da criação de repositórios institucionais. A preocupação com o acesso e a disponibilização da informação científica e tecnológica provoca periodicamente ações voltadas para a melhoria dos processos de geração, armazenamento, acesso e uso moldados pelas tecnologias de informação e comunicação. 
A tecnologia, inegavelmente, pode ser considerada como fator propulsor para a criação dos diversos sistemas que facilitam a produção, acesso e uso da informação. Contudo, a preocupação com a finalidade da produção da informação deve permear os sistemas que se propõem a disponibilizar informação. Isso implica em refletir sobre questões relativas a: Quem produz? O que produz? Em quais condições produz? Qual a contribuição para sociedade? Estas indagações devem permear as reflexões sobre a criação dos novos sistemas de informação, como por exemplo, os repositórios digitais institucionais.

Os repositórios institucionais correspondem a bibliotecas digitais que servem para reunir em um único local toda a produção científica e intelectual de uma instituição, resultando em maior visibilidade e agilidade na recuperação da informação disposta em diversos formatos. Também, fornece estatísticas, facilidade de encontrar informações sobre os produtores e o que está sendo produzido.

No Brasil os repositórios institucionais representam uma solução para o problema de disponibilização da produção científica e intelectual institucional por meio do acesso livre, porém, seu planejamento, implantação e avaliação levantam questões de ordem tecnológica, prática, financeira, cultural e de política institucionais de informação (Silva, 2010).

Os repositórios institucionais enquanto mecanismos de armazenamento de documentos digitais aglutinam e disseminam a produção técnico-científica institucional. Portanto, os repositórios institucionais devem ser organizados de tal forma que permitam ágil acesso e uso das informações. Este artigo propõe estudar os elementos da arquitetura da informação empregada na construção do Repositório Institucional da Universidade de Brasília (RIUnB), usando para tal fim a proposta dos autores Morville e Rosenfeld (2006), e ampliando a análise por meio da compreensão da política institucional e das demandas relacionadas aos usuários.

A escolha do objeto empírico deve-se ao fato do RIUnB ser um dos primeiros repositórios institucionais implantados no Brasil e por ocupar posição destaque no Ranking Web of Repositories (2017).

\section{REPOSITÓRIOS INSTITUCIONAIS}

Com o advento do movimento Open Access muitas iniciativas foram implementadas para fornecer acesso livre e irrestrito a informação científica. No Brasil observa-se a criação de banco de teses e dissertações, periódicos científicos e repositórios temáticos ou institucionais com a finalidade de proporcionar o acesso simultâneo e a preservação da produção científica. 
O repositório institucional, comumente abreviado para ri, representa uma ferramenta importante para a sociedade em geral, pesquisadores e instituições de ensino e pesquisa, pois pressupõe o acesso livre e irrestrito a produção científica e intelectual. Contudo, as instituições ainda enfrentam diversas barreiras de ordem tecnológica, técnica e política. Por exemplo, parcela significativa das instituições de ensino superior não dispõe de recursos financeiros para manter equipe especializada e equipamentos.

Como exemplo de soluções tecnológicas têm-se a adoção de software livre, tal como o Dspace para criação dos repositórios digitais, o Sistema Eletrônico de Editoração de Revistas (SEER) e o Sistema de Organização e Acompanhamento de Conferências (SOAC), os quais resultam da customização do Open Journal Systems (OJS) (Targino et al., 2012). Apesar de a tecnologia facilitar a criação de repositórios, outras soluções ainda estão em andamento para superar as barreiras de caráter político e cultural, como por exemplo, a licença Creative Commons que se constitui em uma estratégia para minimizar questões relativas ao direito autoral.

De Brito et al. (2011) consideram que todos os esforços da produção científica "convergem para o benefício da sociedade esse é também um discurso da ciência. Porém, esses benefícios estão condicionados ao compartilhamento, ampliação, uso e alcance da produção científica através de seu livre acesso e democratização". E assim, a implantação de repositórios institucionais compreende o valor da memória digital para história, economia e cultura nacional (Da Rocha e Galindo, 2009).

Targino et al. (2012) chamam atenção para a diversidade de conteúdo, estruturação e designação de repertórios, ocasionando diferenças em termos de operacionalização e nível de atualização. Como os ri devem atender uma diversidade de usuários e produtores de informação, a arquitetura deve ser pensada e adequada para organizar a informação de forma eficiente e eficaz e promover o acesso. A tríade sugerida por Morville e Rosenfeld (2006) usuários, características de conteúdo e especificidades do contexto já sinaliza que a arquitetura da informação preocupa-se não somente com a variável tecnológica, mas com as políticas e culturais, ou seja, contextuais.

De acordo com Lima Leite (2009), os repositórios podem ser temáticos, quando voltados a comunidades científicas específicas, e institucionais quando voltados à produção intelectual de uma instituição, especialmente universidades e institutos de pesquisa. Para fins desse estudo selecionou-se o termo repositório institucional por abarcar toda produção científica e intelectual de uma instituição de ensino.

Em síntese, o livre acesso à produção intelectual e científica é uma das formas de democratizar a informação e na medida em que indivíduos 
e grupos sociais, caso queiram/necessitem, tenham garantidas as mesmas condições e oportunidades de acesso (De Brito, 2009). Todavia, vale ressaltar que além do acesso, devem também ser garantidas as condições materiais e cognitivas para a apropriação e uso da informação.

\section{Arquitetura DA INFORMAÇÃo Digital}

Possibilitar acesso à informação de maneira estruturada e compreensível, ampliar a navegabilidade nos suportes informacionais e aprimorar a recuperação das informações, essas certamente não são tarefas fáceis de serem realizadas, tanto em meio tradicional quanto e, principalmente, no espaço virtual.

Essas tarefas constituem-se objetivos precípuos da arquitetura da informação, termo surgido na década de 70, mais precisamente no ano de 1976, atribuída ao teórico americano Richard Saul Wurman, com a intenção de solucionar problemas relacionados à informação.

Richard S. Wurman (1997) indicava já naquela época o alvoroço pelo excesso de informações que era refletido na ansiedade dos indivíduos, causando um distanciamento entre a compreensão da informação disponível e o que ela factualmente informava. Segundo Wurman (1997: 16), o objetivo da arquitetura da informação se constituía em "organizar os padrões inerentes dos dados e criar a estrutura ou mapa da informação de forma a permitir que outros encontrem seus próprios caminhos para o conhecimento tornando-o complexo e claro".

No pensamento de van Dijck (2003), o foco da arquitetura da informação é organizar a informação de maneira que os usuários possam encontrar o que procuram e alcançar seus objetivos. Nesse sentido, Batley (2007) comenta que os profissionais da informação devem saber lidar com a informação em suas diversas fontes e formas, de maneira que a informação criada possa ser compartilhada com outras pessoas.

Outras definições apontam para o esclarecimento do termo arquitetura da informação, que conforme o entendimento de Toms e Blades (1999: 247), compreende "a maneira pela qual a informação é categorizada e classificada, armazenada, acessada e exibida, determinando assim, as formas como o usuário poderá encontrar a informação que necessita”.

De forma sistemática, Morville e Rosenfeld (2006: 4) definem a arquitetura da informação como: 
1) O design estrutural do espaço informacional para facilitar a completude das tarefas e o acesso intuitivo ao conteúdo.

2) A combinação dos esquemas de organização, rotulação e navegação dentro de um sistema de informação.

3) A arte e a ciência de estruturar e classificar websites e intranets para ajudar as pessoas a encontrar e gerenciar informação.

4) Uma disciplina emergente e uma comunidade de prática focada em trazer princípios do design e da arquitetura ao espaço digital.

Ademais, na mesma publicação, Morville e Rosenfeld (2006) apregoam a tríade dimensional que deve ser considerada pela arquitetura da informação, onde são abordados os usuários, as características de conteúdo e as especificidades do contexto. $\mathrm{Na}$ dimensão do usuário são incluídas as preocupações com suas necessidades, hábitos e comportamentos. Em relação ao conteúdo, os aspectos se referem ao formato, conteúdo e estrutura da informação. A dimensão do contexto, diz respeito ao uso do sistema de informação que inclui os objetivos do website, a cultura e política organizacional, restrições tecnológicas e localização. Reis (2007: 65) realiza comparação entre essa tríade e as leis de Ranganathan, estabelecidas no campo da Biblioteconomia, afirmando ser uma versão moderna das leis que dispunha em linhas gerais a funcionalidade da biblioteca e a sua função enquanto promotora do conhecimento.

Ainda na perspectiva de Morville e Rosenfeld (2006), eles apresentam quatro sistemas que compõem a arquitetura da informação para websites, intranets e repositórios institucionais, foco desse estudo, sendo eles:

- Sistema de organização: responsável por categorizar a informação e agrupá-la.

- Sistema de navegação: especifica as maneiras de navegar pelo espaço informacional e hipertextual.

- Sistema de rotulação: apregoa as formas de representação e apresentação da informação, definindo signos para cada elemento informativo.

- Sistema de busca: responsável por determinar as perguntas que o usuário poderá fazer e o conjunto de respostas [possível] que irá obter.

Essas definições iniciais alvitram um aprofundamento mais explicativo que será desenvolvido a seguir, buscando a associação entre a arquitetura da informação e os repositórios institucionais, proporcionando uma análise mais consubstanciada. 


\section{ARQUiTETURA DA INFORMAÇÃo EM REPOSITÓRIOS INSTITUCIONAIS}

A arquitetura da informação é constituída conforme apontado na seção anterior por quatro sistemas que foram elaborados por Morville e Rosenfeld (2006). Esses elementos são interdependentes e devem estar apoiados pelas estruturas de representação da informação: metadados, vocabulários controlados e tesauros, que atuarão na organização, descrição, armazenamento e relacionamento das informações (Ferreira de Sousa, 2012).

Dade-Robertson (2011) comenta ainda que os seres humanos procuram dar forma e padrão ao seu ambiente, buscando até mesmo o que se denomina de "arquitetura cognitiva", ao se considerar a forma como o conhecimento pode ser percebido tanto de forma consciente quanto inconsciente.

Para análise dos elementos componentes da arquitetura da informação, será utilizado o Repositório Institucional da Universidade de Brasília. A escolha do RIUnB é justificada pela importância deste repositório que foi demonstrada pela representatividade na publicação Ranking Web de Repositórios del Mundo, em 2013. O ranking é uma iniciativa do laboratório de Cibermetria do Conselho de Investigações Científicas da Espanha (CSIC, 2013). O ranking é realizado desde 2008 e no ano de 2013, o repositório institucional da Universidade de Brasília figurou em $10^{a}$ posição na lista dos melhores repositórios do Brasil. Outra significante justificativa se deve a evolução em volume de publicações do RIUnB, que em setembro de 2008 quando do seu lançamento contava com cerca de 237 publicações disponíveis, atualmente contempla cerca de 13.360 arquivos, dentre eles diferentes tipos como: artigos científicos, livros, capítulos de livros, teses, dissertações, de professores, pesquisadores e discentes da instituição.

O RIUnB constitui-se de um conjunto de serviços oferecidos pela Biblioteca Central da UnB, para a gestão e disseminação da produção científica da universidade. Todos os seus conteúdos estão disponíveis publicamente e, por estarem amplamente acessíveis permitem maior visibilidade e possivelmente maior impacto da produção científica da instituição. Sua missão é armazenar, preservar, divulgar e dar acesso à produção científica da Universidade de Brasília em formato digital. Pretende reunir, em um único local, o conjunto das publicações da UnB (2008).

$\mathrm{Na}$ interface principal do RIUnB estão dispostas informações sobre o repositório, conforme mostra a figura 1: 


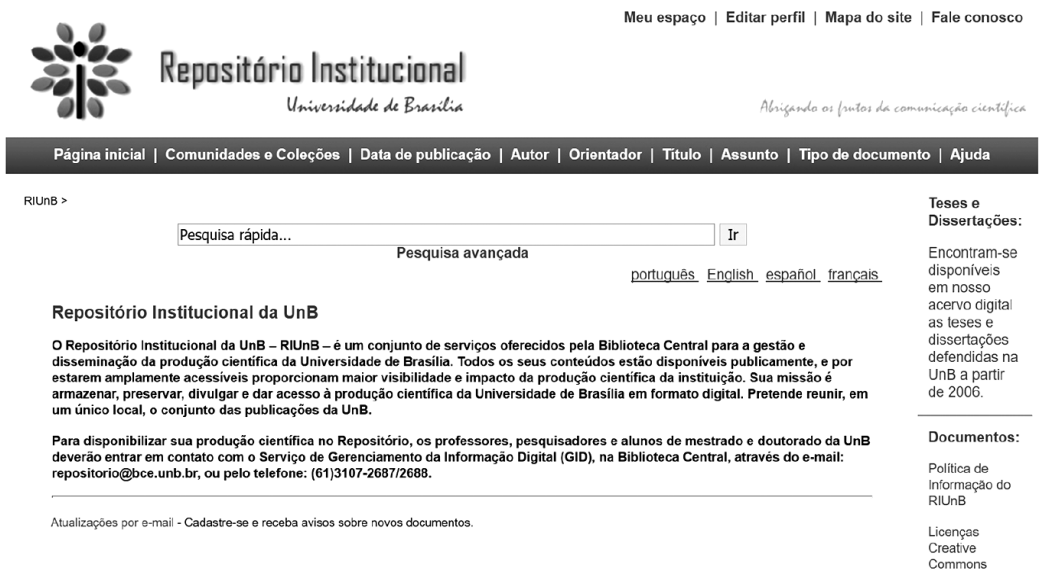

Figura 1. Interface de RIUnB

Fonte: Url:<htpp://repositorio.unb.br>

Esses elementos que compõem a arquitetura da informação foram constituídos com o objetivo de estruturar as informações no ambiente digital, percepcionando as diversas dimensões relacionadas a navegabilidade, organização, recuperação e representação.

\section{Sistema de organização}

Esse sistema se incumbe de tratar da estruturação da informação com a finalidade de facilitar a recuperação de informações. Tem por função definir as regras de classificação e ordenação das informações que serão apresentadas e ao aplicá-las categorizar todos os conteúdos oferecidos (Morville e Rosenfeld, 2006).

A categorização e classificação das informações são tarefas que impõem inúmeras dificuldades e obstáculos aos websites. No caso do repositório analisado, as informações estão categorizadas sob cinco maneiras:

1) Por tipos de arquivo do conteúdo digital: artigos científicos, dissertação, livro, parte de livro ou capítulo de livro, pós-doutorado, relatório, teses, trabalho.

2) Por tópicos, onde apresenta a localização institucional: departamentos, institutos, faculdades, centros, biblioteca central, programas de pós-graduação. 
3) Ordem cronológica através da data de publicação do documento.

4) Ordenamento por autoridade, apresentando as autorias dos trabalhos armazenados no RIUnB.

5) Ainda na perspectiva de organização da informação por autoridade, apresenta-se a possibilidade de busca de informação sobre os orientadores dos trabalhos.

Ampliando a perspectiva de análise do sistema de organização associando-o com o repositório institucional é necessário demonstrar as dificuldades do riunb quanto a organização da informação. Essas dificuldades podem ser classificadas em: ambiguidade, heterogeneidade e diferenças de perspectiva.

No aspecto da ambiguidade os repositórios institucionais enfrentam grande dificuldade quanto ao controle terminológico que possibilite eliminar a ambiguidade que está intrinsecamente relacionada ao processo comunicativo da informação. De acordo com Bustamante (2004: 4), "um mesmo vocábulo pode ter múltiplas interpretações dependendo do contexto onde é apresentado".

Essa multiplicidade de interpretações resulta da ambiguidade e limita o processo de seleção dos rótulos e, além disso, diminui o potencial efetivo de categorização da informação, confundindo o bibliotecário, no momento da inserção e ordenamento das informações.

Outra limitação problemática é a heterogeneidade que desafia o bibliotecário quanto à diversidade de formatos de arquivo. No RIUnB, pode-se perceber a presença predominante do Portable Document Format (PDF), que permite a portabilidade do documento em diversas plataformas de hardwares e softwares. Essa atitude da equipe gestora do repositório demonstra a percepção anterior da dificuldade que viria a ser enfrentada quando da disponibilização de inúmeros arquivos que tornam difícil o gerenciamento da informação nesses ambientes heterogêneos.

A última dificuldade que pode ser atribuída aos repositórios institucionais, são as diferenças de perspectivas, que referem-se a infinidade de visões que se impõem no momento da classificação e categorização das informações a serem inseridas nos repositórios. Cada profissional responsável pela organização das informações nos repositórios pode significá-las de maneiras distintas, conforme sua perspectiva pessoal, sua cultura e sua visão de mundo.

\section{Sistema de navegação}

Conforme afirmou anteriormente e ainda corroborado por Ferreira de Sousa (2012), a interdependência e relacionamento de todos os sistemas é constante. 
Tendo em vista desvendar os caminhos e sendas do ambiente digital, apresenta-se agora o sistema de navegação cuja função é construir os caminhos a serem trilhados pelo usuário.

De maneira conceitual, Vidotti e Sanches (2003: 3) definem o sistema de navegação como sendo "a forma de interação do usuário com o ambiente e com o conteúdo informacional disponível".

O sistema de navegação é um dos principais pontos críticos da arquitetura da informação, pois, envolve a usabilidade da interação dos usuários com o repositório e toda a estrutura informacional que foi planejada pelo sistema de organização da informação. É esse sistema quem vai definir a eficiência da recuperação da informação por meio de estruturas de navegação que irão afetar na permanência ou não do usuário na página do repositório, bem como contribuir para que ele se situe no emaranhado de informações.

Morville e Rosenfeld (2006) dividiram o sistema de navegação em três tipos: global, local, contextual. O sistema de navegação global é aquele menu que está onipresente em todas as páginas do repositório, no caso do RIUnB, existem dois sistemas de navegação global: um composto por página inicial, comunidades e coleções, data de publicação, autor, orientador, título, assunto, tipo de documento, ajuda. E outro na parte superior, que apresenta um menu de navegação global com links para contato e para cadastro/login dos usuários do repositório.

O sistema de navegação local é composto por links que levam a páginas específicas diferentes do sistema de navegação global, que não estão presentes em todas as páginas. No RIUnB a navegação local é plural, abrange links para facilitar a recuperação de informações por meio de categorias já explicitadas anteriormente nesse estudo.

O sistema de navegação contextual são links que possibilitam a ampliação da navegabilidade, são conhecidos por expressões como: "ver mais"; "consulte também"; "artigos semelhantes". Esse tipo de sistema de navegação permite que o usuário associe com maior facilidade as informações, e ainda amplia a curiosidade por outras informações que poderiam passar despercebidas no momento da navegação. Pode-se considerar como sistema de navegação contextual os descritores sob a forma de hyperlinks que descrevem os documentos digitais associando-os a outros que foram categorizados pelo mesmo assunto.

Outro elemento importante que deve ser ressaltado quanto ao sistema de navegação em repositórios institucionais, é a navegação estrutural ou também conhecida sob o termo inglês BreadCrumb que como aponta Reis (2007: 95) trata-se de "uma lista de elementos (links) separados por algum 
caractere (normalmente $>$, : ou $\mid$ ) ou gráfico (normalmente uma seta para a direita). Tem a intenção de informar o usuário onde ele se encontra na estrutura do website e prover links para os níveis hierárquicos imediatamente superiores".

No RIUnB, o BreadCrumb detalha o caminho que o usuário percorreu a partir do momento em que inicia a navegação pelos links disponíveis no repositório, passando pelas categorias de tópicos institucionais. Entretanto, esse elemento de navegação só consta em estruturas de navegação internas do repositório, que englobam as categorias onde "encontram-se os documentos".

\section{Sistema de rotulação}

$\mathrm{O}$ ato de rotular assim como o de categorizar informações é invariavelmente uma necessidade do ser humano. Rotular informações é uma perigosa aventura no mundo digital, ainda mais quando se enfrenta um amplo espaço onde o dinamismo informacional é frequente. Nos websites rótulos são empregados nos títulos de páginas, nas opções do sistema de navegação, nos links contextualizados das páginas e nos metadados utilizados na indexação das páginas para o sistema de busca (Morville e Rosenfeld, 2006).

Nos repositórios institucionais a rotulação pode ser textual ou não textual, imagética. A rotulação textual é aquela composta por uma ou mais palavras. Já a rotulação não textual é onde se atribui imagens para representar os conceitos (Reis, 2007).

Um dos problemas que permeiam os sistemas de rotulação está relacionado ao idioma, a sua subjetividade e ambiguidade que confluem para limitar a possibilidade de aprimoramento da rotulação das informações que irão guiar os usuários pelos repositórios.

Além disso, outras dificuldades são inerentes a esse sistema, por exemplo, as diferentes formas de rotular uma informação que difere de usuário para usuário, o que limita a sua capacidade de navegação e de usabilidade do repositório. No RIUnB a rotulação é feita de maneira textual, onde são apresentadas as categorias que foram indicadas na seção que analisa o sistema de organização do repositório.

\section{Sistema de busca}

Outro ponto crucial da arquitetura da informação é a busca. Morville e Rosenfeld (2006) esclarecem que esse sistema deve ser muito bem planejado antes de se incluir em um website, pensamento do qual esse estudo corrobora, porém, voltando a atenção para os repositórios institucionais, que reúnem 
diversos tipos de informações, organizadas sob distintas formas, tornando a busca um elemento essencial.

O sistema de busca auxilia a localização e o acesso direto às informações armazenadas em um website (Gay, 2009). Para realizar a recuperação das informações utilizando o sistema de busca, é necessário realizar anteriormente a representação dessa informação. A representação temática diz respeito à atividade de descrever e identificar um documento de acordo com o seu assunto. A representação descritiva é a codificação de informações contidas em documentos, de forma que permita a interseção com outras informações que se encontram na mesma base de dados e, informações solicitadas pelos usuários.

O mecanismo de busca do RIUnB está localizado na parte central superior da interface principal do repositório e a sua estruturação está direcionada à realização de buscas simples e avançadas. A busca simples, também denominada no repositório estudado como pesquisa rápida, permite a pesquisa por um único termo e possibilita recuperar informações em duas opções: em todo o repositório ou em departamentos específicos da UnB. Já a pesquisa avançada, oferece a possibilidade de pesquisar informações que constem nas categorias temáticas descritivas de autor, título, assunto, língua do documento, resumo, séries e identificador (número atribuído pelo repositório aos documentos armazenados) conforme ilustra a figura 2:

Pesquisa: Todo o repositório

Tipo de pesquisa: Pesquisar por:

Pesquisa geral
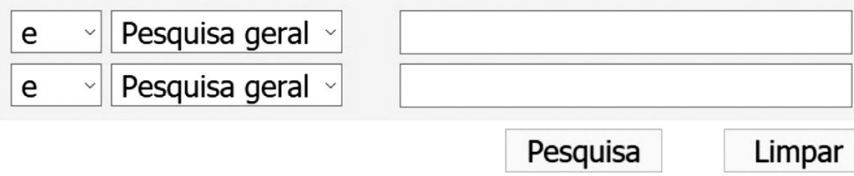

Figura 1. Interface de RIUnB

Fonte: Url::htpp://repositorio.unb.br>

A apresentação dos resultados no RIUnB oferece a opção de organizar os resultados da busca de maneira crescente ou decrescente, por ordenamento cronológico (data de submissão ou publicação do documento), ordenamento de relevância, além da quantidade de registros a serem exibidos. 


\section{Demandas de informação dos usuários}

As necessidades das comunidades de usuários devem ser consideradas em todo o momento de planejamento da arquitetura da informação dos repositórios institucionais. A determinação dos serviços que serão oferecidos pelo repositório e o seu funcionamento deverão ser objeto de análise prévia para atendimento das necessidades dos usuários. Lima Leite (2009) ao citar Crow enfatiza que a acomodação das necessidades e percepções da comunidade e a demonstração de sua relevância para a satisfação dessas necessidades devem ser um componente central das políticas de conteúdo, planos de implementação e de marketing do repositório institucional.

A pluralidade de usuários pode se constituir em um problema a ser enfrentado pelo arquiteto da informação de repositórios institucionais, pois esses repositórios contemplam desde usuários com perfil científico (docentes, discentes, pesquisadores, etc.) a usuários com perfil técnico (administradores, técnico-administrativos, bibliotecários, etc.), e indivíduos que não fazem parte da comunidade científica.

As necessidades dos usuários de repositórios institucionais, principalmente de universidades devem ser identificadas a partir da prospecção de demandas de informações com os departamentos acadêmicos, programas de pós-graduação, gestores das bibliotecas que integram a IES. Essa prospecção de demandas permitirá a primeira visão sobre que informação deverá ser armazenada e disponibilizada pelo repositório. Outra avaliação de demandas deve ser realizada com os usuários do repositório antes de desenvolvê-lo, se possível, e durante o processo de funcionamento desse, mantendo contato permanente para avaliação constante.

No caso do RIUnB, dentro do repositório existe um link que diz respeito ao "fale conosco" onde os usuários podem fazer sugestões e críticas. Outro espaço mais específico é o "meu espaço", onde os usuários podem cadastrar-se e ter acesso a submissão de itens a coleção a qual está cadastrado, mediante autorização prévia da equipe do RIUnB. Podem também, a partir de informações cadastradas que descrevem o seu perfil de interesse, receber alertas enviados por email pelo RIUnB que informam a inserção de novos documentos que tratam de assuntos de interesse.

\section{Política institucional}

A política institucional é outro fator influente no processo de arquitetura da informação em repositórios institucionais de IES. A política confere um conjunto de regras que institui, principalmente, a implantação, manutenção, 
alimentação de informações e hospedagem do repositório, e as responsabilidades e atribuições dos indivíduos.

Além disso, deverão ser designadas as formas pelas quais os usuários poderão acessar os conteúdos do repositório, acesso livre ou acesso restrito a instituição. No caso do RIUnB, no ano de 2013 foi aprovada a resolução de $n^{\circ}$ 101/2013 (UnB, 2013) que regulamenta a política de informação do repositório institucional da Universidade de Brasília. Dentre as decisões, estão: a) a atribuição da responsabilidade pelo desenvolvimento, implantação, manutenção, hospedagem e alimentação do RIUnB a Biblioteca Central; $b$ ) a determinação de livre acesso, tanto no contexto nacional quanto no internacional; c) capacidade de integração com sistemas nacionais e internacionais, observando-se o uso de padrões e protocolos de integração, em especial aqueles definidos no modelo Open Archives; d) funcionamento em plataforma desenvolvida em software livre, em consonância com os princípios do acesso aberto.

Os pontos em questão demonstram a funcionalidade e importância do estabelecimento de uma política institucional que regulamente o repositório institucional perpassando por todas as fases. Um fator importante que foi destacado nessa resolução do RIUnB é o acesso aberto a publicações científicas, importante ação que contribui para o desenvolvimento da informação científica, contemplando a preservação, armazenamento, organização e gerenciamento da produção científica produzida pela instituição.

\section{CONSIDERAÇÕES FINAIS}

De forma geral o RIUnB apresenta compatibilidade com alguns sistemas que compõem a arquitetura da informação propostos por Morville e Rosenfeld (2006), dentre eles estão o sistema de organização, navegação e rotulação. No primeiro sistema, a organização das informações no RIUnB apresenta uma intensidade de categorias que permitem aos usuários localizá-las rapidamente. Outro ponto positivo é a ausência de heterogeneidade nos formatos em que estão armazenadas as informações, o que confere ao RIUnB a facilidade de gerenciar melhor as informações.

Já com relação à navegação, embora o sistema global apareça em todas as páginas do repositório, foi identificado que não existe o detalhamento do caminho percorrido pelo usuário até acessar a informação desejada, por exemplo, se usuário acessar o link Comunidades e Coleções, e selecionar a comunidade "Centro de Desenvolvimento sustentável" e realizar uma pesquisa por assunto, a página que apresenta os resultados não informa o caminho realizado até então. Recomenda-se a utilização da navegação estrutural BreadCrumb para essa categoria. 
Porém, algumas limitações do repositório foram identificadas quanto ao atendimento às demandas de informação dos usuários e sistema de busca. O RIUnB disponibiliza tutoriais que explicam como se cadastrar, navegar e pesquisar no repositório, e respostas às perguntas mais frequentes. Contudo, o tutorial não contempla a explicação da composição de cada comunidade, o que dificulta a recuperação da informação por parte do usuário na comunidade desejada. Recomenda-se a utilização de rótulo de ajuda que acompanhe cada comunidade, explicando o conteúdo que poderá ser encontrado em cada uma. Além disso, a inclusão dos contatos telefônicos e email no link "Fale conosco", o qual apresenta apenas local para inserir e-mail e comentários.

Em relação ao sistema de busca, a limitação identificada está relacionada a execução da pesquisa avançada que oferece poucos recursos para o usuário, somente uma busca com a utilização de operadores booleanos (and, not, or), que apresenta a inabilidade em ordenar os documentos resultantes de uma busca. Não apresenta opções de refinamento dos resultados, apenas alternativas para ordenar por relevância ou quantidade.

O RIUnB apresenta insuficiência de recursos de acessibilidade e usabilidade, pois apenas para ilustrar, o RI não apresenta recursos para aumentar e diminuir letras, alterar cor de fundo e recurso de leitura de tela para deficiente visual na interface.

A equipe que administra o RIUnB também deverá preocupar-se com os desafios contemporâneos de acesso à informação, como por exemplo, desenvolver a arquitetura da informação para dispositivos móveis. A questão é como proporcionar ao usuário o uso do repositório institucional em dispositivo móvel, de forma a possibilitar a recuperação da informação de forma ágil, já que atualmente para o usuário acessar a "homepage" (que foi projetada para computadores) por meio de um dispositivo móvel, enfrentará dificuldades referentes ao volume de informações disponibilizadas, e de tempo para carregar a página e acessar o documento em formato específico para esse tipo de dispositivo.

Outro desafio é como apresentar os diversos formatos da informação em uma única tela, proporcionando assim, visualizar a imagem, som e texto referente a um mesmo assunto numa mesma tela tanto para computadores como para dispositivos móveis.

Puntoni (2013), coordenador da Biblioteca Brasiliana, afirma que no Brasil já há estudos para o desenvolvimento de ferramentas que possibilite o acesso aos repositórios em dispositivos móveis, atendendo assim a públicos ou segmentos de usuários que utilizam destes dispositivos para acessar 
e produzir informações. Assim, novos desafios referentes ao acesso são postos diariamente às equipes gestoras dos repositórios institucionais.

Por fim, pode-se afirmar que essa análise propicia aos repositórios institucionais, uma reflexão quanto à importância da estruturação dos repositórios conforme as diretrizes apregoadas pela arquitetura da informação, possibilitando verificar deficiências e aperfeiçoar elementos que estejam em congruência com seus princípios.

\section{REFERÊNCIAS}

Batley, Sue. 2007. Information Architecture for Information Professionals. Oxford: Chandos.

Borba, Vildeane da Rocha; Galindo, Marcos. 2009. "Preservação digital: modelo orientador para o bdtd/ufpe". Encontro nacional de pesquisa em ciência da informação 10. Anais... João Pessoa.

Brito Lima, Celly de. 2009. "Identidades afrodescendentes: acesso e democratização da informação na cibercultura”. Mestrado em Ciência da informação - Universidade Federal da Paraíba, João Pessoa, dissertação, 117 f.

Brito Lima, Celly de, Edilene Maria Silva, Gimene Cunha Rodrigues. 2011. "Pesquisa-ação na implantação do repositório institucional da ufpe". Conferência sobre tecnologia, cultura e memória. Anais eletrônicos... Recife, Disponível em: <http:// www.liber.ufpe.br/ctcm/anais/anais_ctcm/17_pesq_reposit_UFPE.pdf $>$, consultado em 30 maio 2013.

Bustamante, Antônio M. de Oca Sánchez. 2004. "Arquitectura de información y usabilidad: nociones básicas para los professionales de la información”. Disponível em: <http://bit.ly/1cH8Cou>, consultado em 7 nov. 2013.

Consejo de Investigaciones Científicas de España (csic). 2013. "Ranking Web de Repositórios del Mundo”. Julio. Disponível em: <http://bit.ly/14UnLs1>, consultado em 18 nov. 2013.

Dade-Robertson, Martyn. 2011. The architecture of information: Architecture, interaction design and the patterning of digital information. Londres: Routledge.

Gay Fantinel, Rosemary. 2009. "Bibliotecas digitais em Arquitetura e Urbanismo: um estudo sobre arquitetura da informação digital”. Natal: Mestrado em Arquitetura e Urbanismo Programa de Pós-graduação em Arquitetura e Urbanismo, Universidade Federal do Rio Grande do Norte, dissertação, 268 f.

Lima Leite, Fernando César. 2009. Como gerenciar e ampliar a visibilidade da informação cientifica brasileira: repositórios institucionais de acesso aberto. Brasília: ibict.

Morville, Peter; Rosenfeld, Lou. "Information Architecture for the World Wide Web: Designing Large-Scale Web Sites”. 3. ed. Sebastopol, CA: O’Reilly \& Associates, 2006.

Puntoni, Pedro. 2013. "Biblioteca Brasiliana-USP". Conferência sobre tecnologia, ciência e memória - CTCM, 2, Recife, Conferência de abertura.

Ranking Web of Repositories (RWR). 2017. http://repositories.webometrics.info/en/ Americas/Latin_America. 
Reis, Guilhermo Almeida dos. "Centrando a Arquitetura de Informação no usuário”. 2007. 250 f. Dissertação (Mestrado em Ciência da Informação) - Escola de Comunicações e Artes, Universidade de São Paulo, São Paulo, 2007.

Silva, Edilene Maria. 2010. Informação em ciência e tecnologia: políticas e estratégias. Recife: Nectar.

Ferreira de Sousa, Marckson Roberto. 2012. "O acesso a informações e a contribuição da arquitetura da informação, usabilidade e acessibilidade”. Inf. E Soc. 22 (num. especial): 65-76. Est., João Pessoa. Disponível em: <http://bit.ly/17fYp$\mathrm{Nm}>$, consultado em 07 nov. 2013.

Targino, Maria das Graças, Joana Coeli Ribeiro Garcia, Maria José Rodrigues Paiva. 2012. "Repositórios institucionais brasileiros: entre o sonho e a realidade". Conferencia Internacional BIREDIAL-ISTEC, Cidade Barranquilla, Col. Disponível em <http://eventos.uninorte.edu.co/index.php/biredial/biredial2012/paper/ view/360>, consultado em 02 nov. 2013

Toms, E.G and Ruth Blades. 1999. "Information architecture and web site design". Feliciter 45 (4). Disponível em <http://www.cla.ca/feliceter/45-4/column2. htm>, consultado em 7 nov. 2013.

Universidade de Brasília. 2013. "Resolução da Reitoria n¹01/2013”. Disponível em $<$ http://bit.ly/17IK4ta>, consultado em 8 nov. 2013.

Universidade de Brasília. 2008. "RIUnB Sobre Repositório Institucional da unb". Disponível em <http://bit.ly/17bl2x6>, consultado em 7 nov. 2013.

Van Dijck, Peter. 2003. Information architecture for designers: structuring websites for business success. Hove, UK: Rotovision.

Vidotti, Silvana Aparecida Borsetti Gregorio e S.A.S. Sanches. 2003. "Arquitetura da informação em websites”. Disponível em <http://bit.ly/1c3ikfR>, consultado em 7 nov. 2013.

Wurman, Richard Saul. 1997. Information architects. Nueva York: Graphis.

Para citar este texto:

Silva, Edilene Maria, Marckson Roberto Ferreira de Sousa y Samuel Alves Monteiro. 2018. "Arquitetura da informação em repositórios institucionais: desafios e perspectivas". Investigación Bibliotecológica: archivonomía, bibliotecología e información 32 (76): 45-61.

http://dx.doi.org/10.22201/iibi.24488321xe.2018.76.57975 